\title{
Local Government Unit (LGU) and Academe Partnership for Responsive e-Disaster Systems
}

\author{
Maria Victoria Pineda \\ College of Computer Studies, De La Salle University, Manila, Philippines \\ e-mail: mavic.pineda@delasalle.ph
}

\begin{abstract}
Many developing countries have ventured to disaster management programs in the desire to minimize if not, eradicate disaster vulnerabilities and improve the coping skills of the people. Many ICT solutions have been developed and unfortunately, many of these are just one-time big-time solutions. Usability, transparency, costs of the systems and sustainability had become some of the issues.

It is in this light that a working model of partnership between the academe and the local government unit ( $L G U)$ as a community is shaped. The academe takes the initiative in this endeavor. The tie-up is intended to support the LGU to become more responsive in managing its resources and addressing the needs of its constituents.

The paper imparts the experience of the academe's ICT project incubation for a community and further leads to a meaningful cooperation with the LGU. The ICT project developed is a flood prediction and mapping system for the province of Bulacan in the Luzon island of the Philippines.
\end{abstract}

Keywords: e-Disaster system, mitigation system, academe-LGU partnership

\section{Introduction}

In crafting disaster management programs to support communities in developing countries, climate, geography, population and economic conditions are the primary factors considered. But more than these, a workable disaster management program should include research, education and training aimed at eliminating vulnerabilities (Watanabe, no date). Further, "analysis of resources, socioanthropological factors and capacity for governance” that push or pull the programs must be considered as well (Watanabe, no date).

To eliminate vulnerabilities may sound too good to be true. But, in the advent of information communications technologies (ICT) and other useful web solutions, the convergence of research, education and training and ICT solutions with systematic methods, resources and capacity analysis, elimination of risks and vulnerabilities would be possible.

ICT drives our lives nowadays. ICT also has become a cornerstone of progress in many nations including the Philippines. It is a "dominant and vibrant force that led to diffusion and application in a nation's development” (Mokhtar, 2007). Likewise the creative and pragmatic use of ICT can effectively deliver a pure public service that will benefit all societal stakeholders (Pineda, 2010). 
In the recent report of Gonzales and Vanegas (2010) in South America, ICTs are used for data delivery, public awareness and education, capacity building, public involvement and institutional strengthening within disaster mitigation.

Experiences of developed and developing countries are incomparable as far as resource management and capacity-building are the trepidations. Hence, the goal of this paper is to present a workable model that fuses research, ICT and strong cooperation between the government and the academe for a sustainable effort of developing disaster management systems and applications in developing countries.

This paper also presents a recent incubation project, the Sawatain, a flood prediction system, intended to be a tool for disaster mitigation and preparedness. The paper imparts how a partnership was borne out of the efforts of the academe to support an LGU and the LGU in return adhering to the invitation.

\section{CITe4D's Community-based Disaster Risk Reduction Research}

The Center for ICT for Development (CITe4D) of the College of Computer Studies of De La Salle University has ventured for the past years on developing web-based disaster management systems. The CITe4D started to study and design disaster response systems for the leading government agencies during the first phase. In the second phase, CITe4D focused on disaster mitigation systems as hazard mitigation is a very strategic approach to harm reduction in developing countries (Doberstein, 2010). The approach has been to develop community-based disaster risk reduction functional prototypes. CITe4D at present has several on-going LGU partnerships and one of them is with Bulacan.

Further, Rahman (2002) and Gaillard and Le Masson (2007) have agreeing views that an effective approach to supporting the coping competencies of the people is through community-based disaster preparedness and risk reduction. Most developing nations adhere to the same idea. In the Philippines, it was last May 2010 when the Philippine Disaster Risk Reduction and Management Law (RA10121) was passed. The law now encompasses “disaster risk reduction, preparedness and mitigation” from the former emphasis on disaster response and recovery (Sy, 2010). While it was only recent the Philippines finally braced a national policy on disaster risk reduction, many local communities have exerted efforts on proactive ways of community-based disaster preparedness, one of which is the Bulacan province, considered as the "Most Disaster-Prepared Province in Central Luzon” (Velez, 2010). 


\section{Sawatain, the Flood Prediction System for Bulacan}

In September 2009, Typhoon Ketsana, brought an estimated $45 \mathrm{~cm}$ of rainfall in 24 hours, equivalent to a month's rainfall in the monsoon season and affected over $2.2 \mathrm{M}$ people because of flooding (COE-DMHA, 2009). This deluge motivated the development of a mitigation system. The scope of the study focused on the Bulacan province.

Bulacan is a suburban province situated north of Metro Manila. It has 21 municipalities, 3 cities and 569 barangays. The topography is from level to rugged terrain with many streams and large rivers providing water and hydroelectric power. There are 3 dams--Angat, Ipo and Bustos. Angat Dam provides the greater portion of water supply in Metro Manila as well as providing electrification in the whole of Bulacan (Provincial Government of Bulacan, 2007).

Sawatain (came from the Tagalog word "to mitigate" or "to stop") is a web-based flood prediction system which focuses on mapping the flood hazard or affected areas in the province of Bulacan. The stakeholders and important agencies involved in the disaster management workflow are able to study the effects and impacts of an incoming typhoon by district or the whole province through visual mapping.

The Sawatain system aims to predict the risk level of the municipalities in the province though simulations of the impact of rainfall in the province. This is done effectively with the simulation versioning feature of the system. The versioning can be done before the actual rainfall or when the rainfall reaches the area of responsibility. Other variables that were considered are the dam outflow, level of terrain and the projected number of hours of rainfall. It also predicts the number of people and families which will be affected in each town and informs important agencies such as the local government units, the office of the governor, and other provincial agencies. The system provides an

effective alternate method of early warning through the web system and mobile/SMS (short messaging system) facilities.

The system supports the decision-making process of the Bulacan Provincial Disaster Management Office and the office of the governor by providing on-time and reliable reports. The expected flooded areas and evacuation centers also become transparent to the citizens with the use of the Google maps.

It was developed using open source development tools making it a very cost-effective solution and encouraging open development. The system can be readily modified to cater to other specific needs 
of Bulacan. The system developers had to conduct their data gathering and interviews with the Provincial Disaster Management Office, the PAG-ASA weather bureau, the National Power Corporation, and the Angat Dam experts.

\section{Sample Screens}

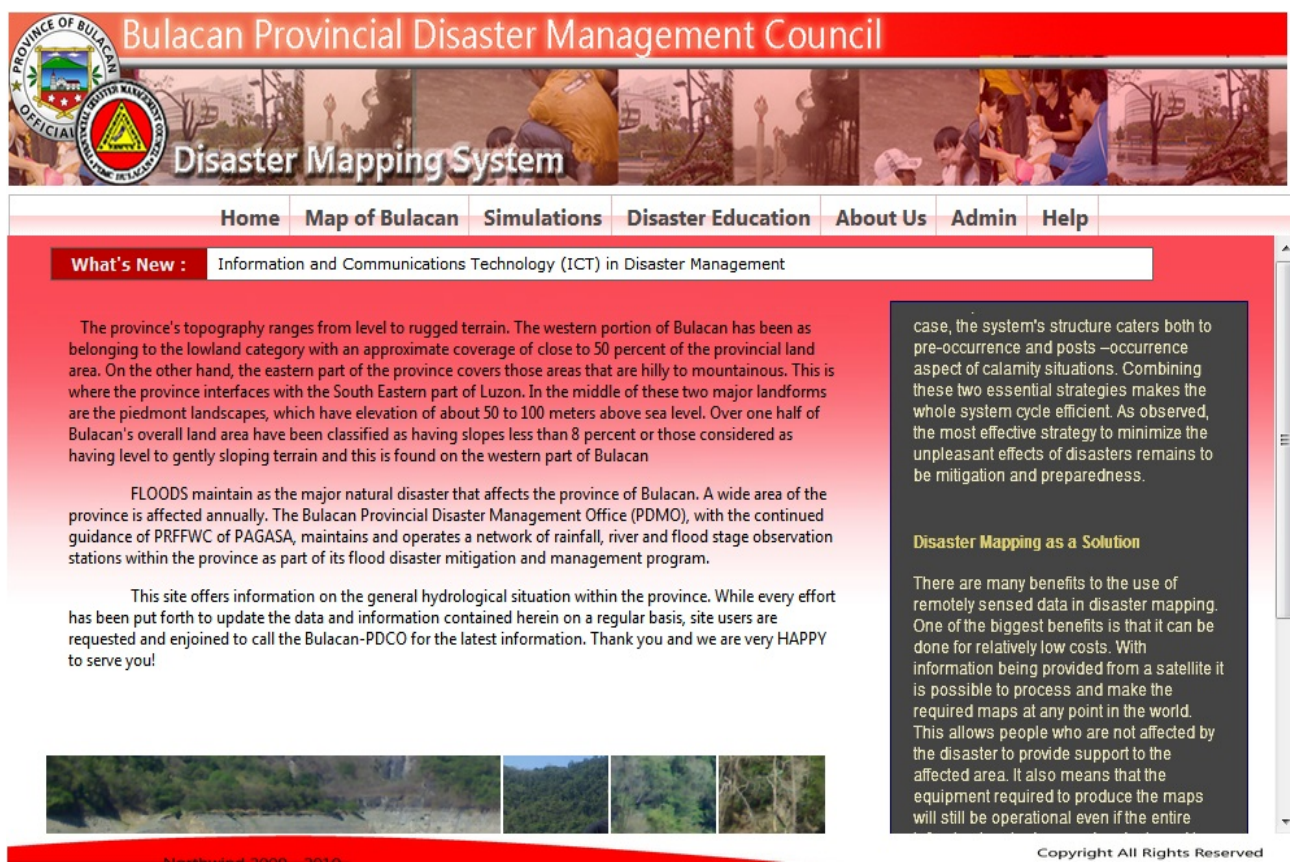

Figure 1. The Sawatain Main Screen
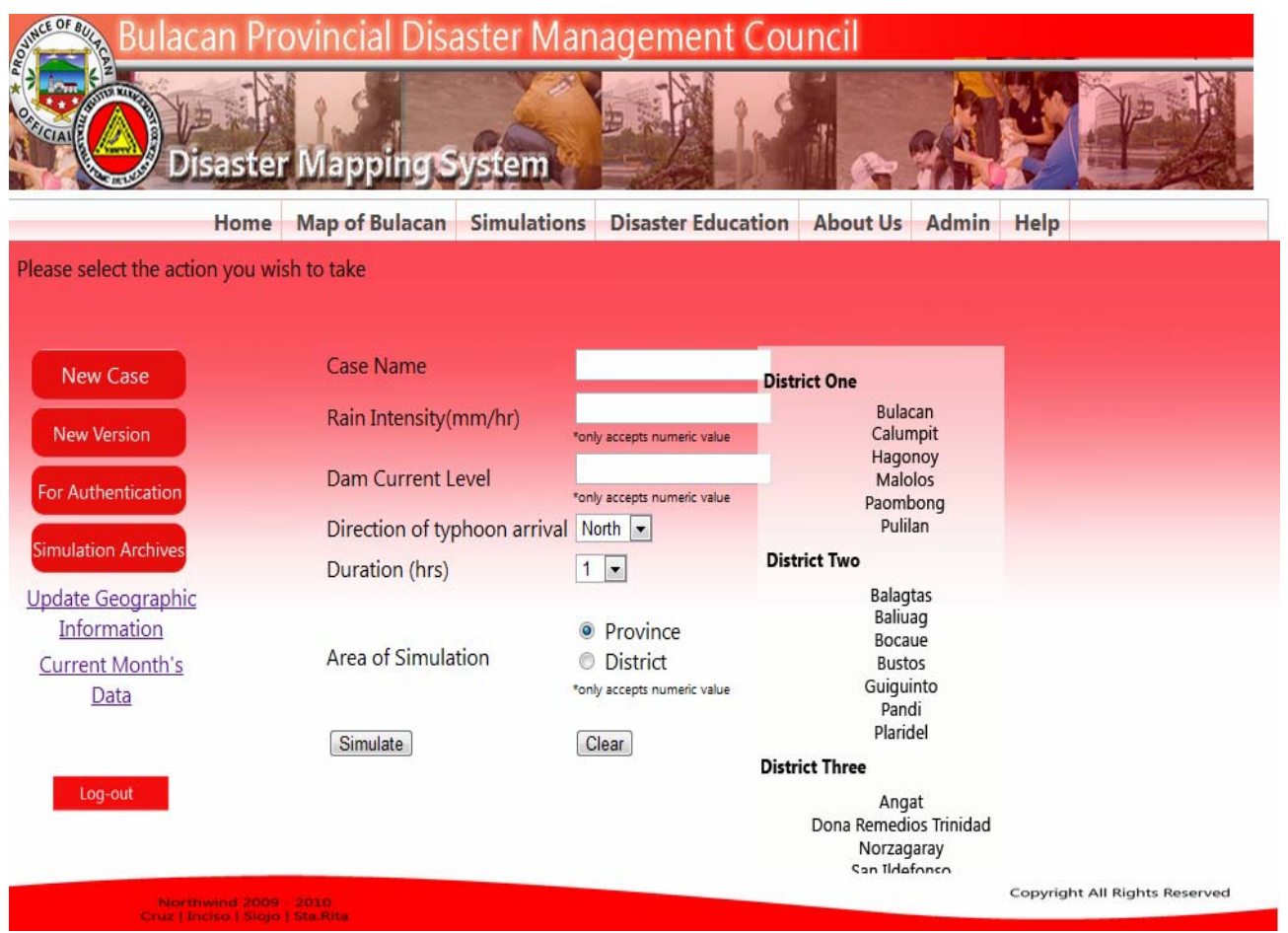

Figure 2. The Sawatain Simulation Screen 


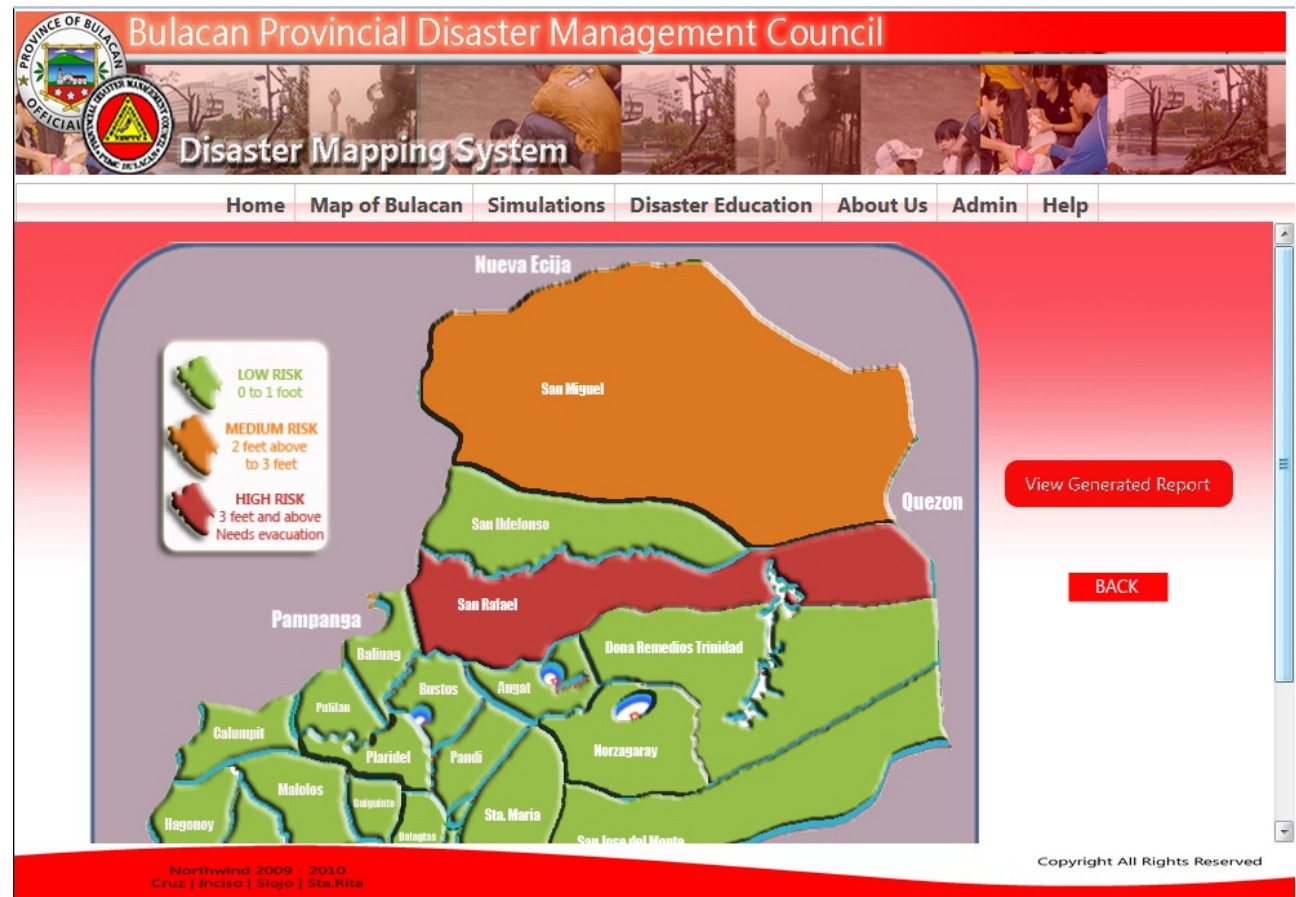

Figure 3. The Sawatain Sample Map after the simulation
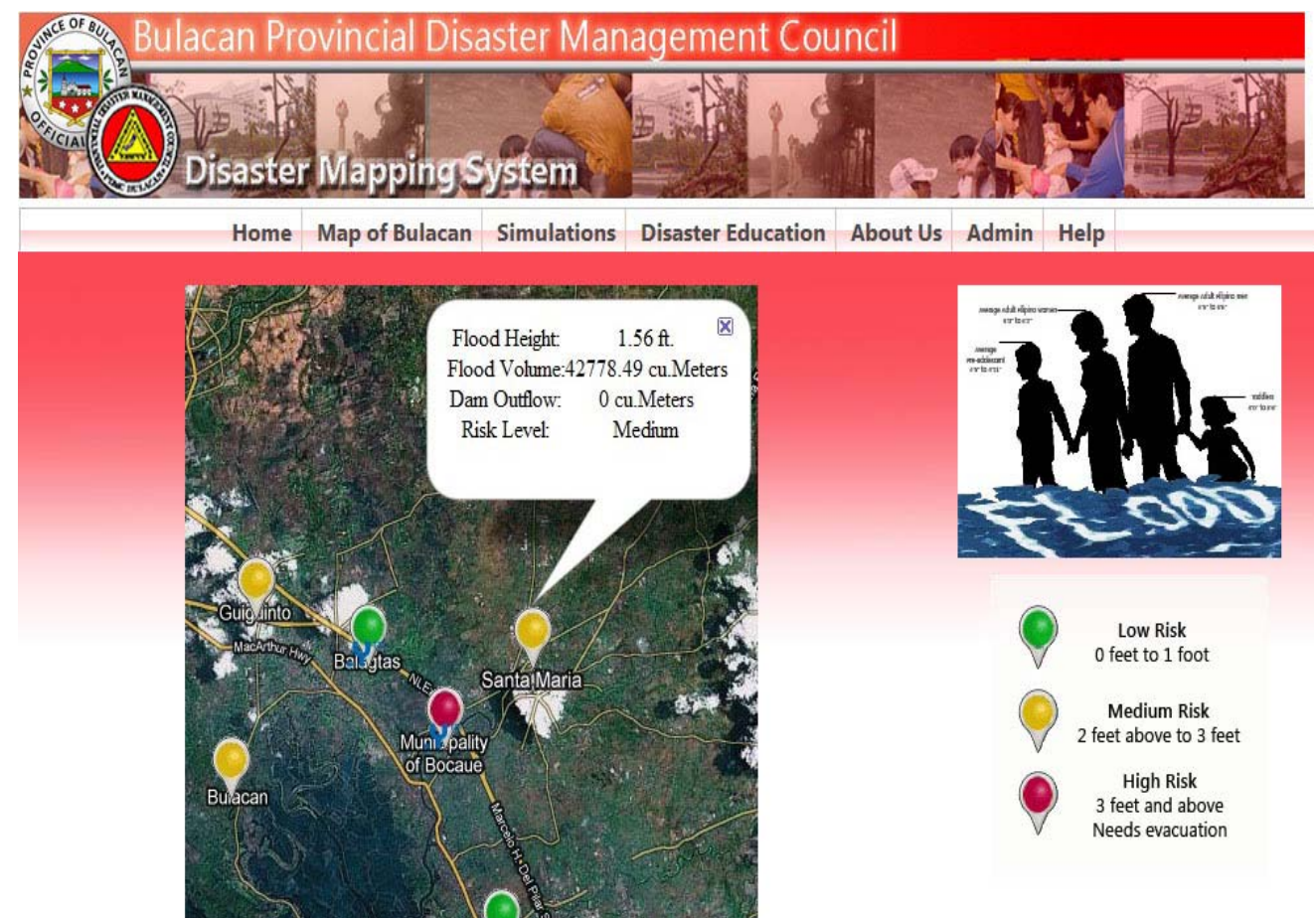

Figure 4. The Sawatain Google map for reference of the citizens in terms of hazards and evacuation centers.

\section{Proposed LGU-Academe Partnership Model}

According to Bildan (2003) "an affected community has a variety of urgent needs that can be responded to in a timely and appropriate manner”. Hence action and resolve plans would be a concerted effort by the stakeholders such as the civil society, the government and the private sector. 
This is where the academe would come in. The academe, especially the higher education institutions, can spearhead and initiate meaningful partnerships with the local communities or the LGUs in crafting ICT solutions that will enhance disaster mitigation and preparedness. Based on the CITe4D experience, there is a very good opportunity of designing systems that cater to the requirements of the community and developing cost-effective solutions.

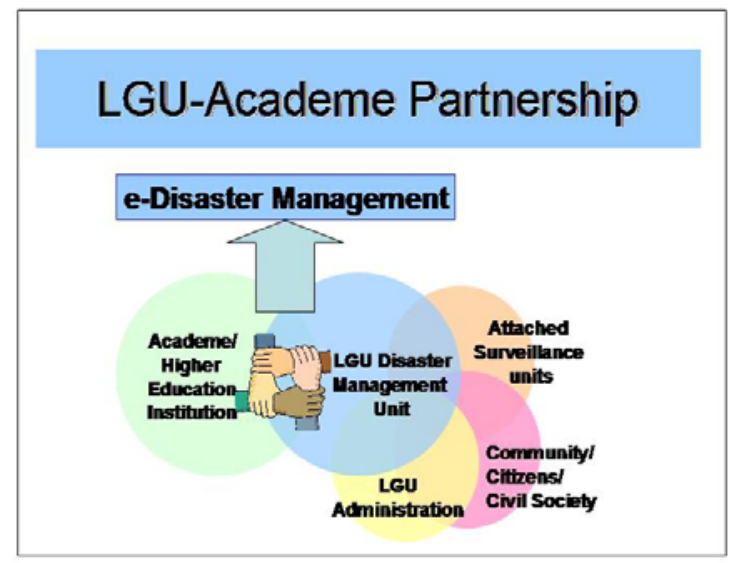

Figure 5. The LGU-Academe Partnership Model

In the LGU-Academe Partnership model above, the major involvement lies on the academe and the LGU's disaster management unit. This LGU disaster management unit can be at the provincial level or at the municipal level.

The LGU disaster management unit is in-charge of directing all the activities and efforts initiated by the partnership. Regular consultation with the academe unit is vital.

The academe shall interface with the other entities such as the LGU office, the community and including the attached surveillance agencies like the weather bureau, the water resources administration or the local police. The role of the academe is to capture the workflow and coordination processes of the different entities, determine the user requirements, and design a suitable infrastructure and IT solution given the present resources and sustainability capabilities of the LGU.

The academe unit, equipped with strong ICT competency, is in-charge of the systems' feasibility or prototyping study, development of the project details and terms of references and the whole project management and development.

The model is intended to be a continuing partnership. This is enhanced by active participation and presence of a change champion. A change champion is somebody who has great faith in nation building, exerts high level of efforts to see the delivery of excellent systems and programs. To cite, in the stages of the Sawatain project, the weather bureau scientist is the change champion. 


\section{The Bulacan Partnership Experience}

In most of the CITe4D's community engagement, the research center took the initiative to be involved, to do research and get to know the community together with the Information Technology students and the research advisers. Communities welcome partnership with the academe. Communities are aware of their own weaknesses and they are typically willing to invest time to create solutions and be supported by the academe.

The Bulacan is one of the on-going tie-up of the research center with the community. Bulacan stakeholders are confident it is a win-win cooperation with the academe. The disaster management office of Bulacan was grateful to see that the flood prediction system developed was able to capture the work processes and the methods they employ in the province. They also commended the appreciation of the web system design that exhibits the Bulacan ways and traditions.

Plans are underway to further improve the Sawatain system, integrate it with the existing digital rain gauges of the disaster management unit for more accurate reports.

\section{Conclusions}

Mango and Rafisura (2007) posted that the main challenge for developing countries would be to cascade the national policies on disaster management to local disaster preparedness and mitigation plans. But with the available skills and competencies from the academe and the expertise of the LGUs, the direction now is actualizing all the mitigation and preparedness plans.

There are two faces in developing e-disaster system solutions. First is the need to determine the real needs and the resources of the community. A community has its own topographical characteristics and its own strategies in dealing with its hazards. These socio-anthropological factors are underscored for in-depth scrutiny.

The other face indicates the localized systems approach to ICT solutions development is imperative. This means commercial ICT disaster mitigation solutions that are costly or may not be customizable or are not be able to capture the work processes and methods of the community may not effectively serve the community. Localized approach still best serves the purpose.

The higher education institutions specializing in ICT that have ventured in many community projects have the necessary expertise in development. The LGU-Academe Partnership model brings 
about a nurturing cooperation to develop better e-disaster systems, increase public awareness and citizen involvement, participation of surveillance agencies and better disaster governance from the local government units.

The close tie-up does not happen overnight. It is like planting a tree. The tree is nurtured with water, sun and much care to see it bear fruit. This is what it takes to have a sustainable partnership.

\section{Acknowledgment}

This paper would like to acknowledge the Sawatain system developers, namely, Nicole Inciso, FJ Sta. Rita, Juno Sioco and Raymund Cruz. This paper would also like to express appreciation to the DLSU-AdRIC and CITe4D, the research centers of the college.

\section{References}

Bildan, L. (2003). Disaster management is Southeast Asia an overview, Asian Disaster Preparedness Center.

COE-DMHA. (2009). Tropical storm Ketsana Update September 30, 2009, Center for Excellence in Disaster Management and Humanitarian Assistance. Retrieved from http://www.coedmha.org/apdr.

Doberstein, B. (2010). Adaptive hazard mitigation: the theory and practice of responding to environmental change-driven disasters, Proceedings of the ICSBE 2010 International Conference on Sustainable Built Environment with the theme Enhancing Disaster Mitigation and Prevention, May 25-27, 2010, Jogjakarta, Indonesia.

Gaillard, J.C., and Le Masson, V. (2007). Traditional societies' response to volcanic hazards in the Philippines. Mountain Research and Development, vol. 27(4), pp. 313-317.

Gonzalez, P., and Vanegaz, W. (2010). Applying information and communication technology for disaster mitigation in the Central American Isthmus, Final Technical Report, IDRC Project Number 104410-001, July 2010.

Magno, F., and Rafisura, K.M. (2007). Developing research education agenda on disaster preparedness, Workshop: Strengthening local government capacity for disaster preparedness in Asia, Manila, Philippines, October 8-9, 2007.

Mokhtar, K.S. (2007). ICT and distance education: towards local disaster preparedness in Malaysia, International Workshop and Book Project, Strengthening local government capacity for disaster preparedness in Asia, Manila, Philippines, October 8-9, 2007. 
Pineda, M.V. (2010). A perfect storm, a perfect disaster, and the challenge to responsive disaster management systems, Proceedings of the ICSBE 2010 International Conference on Sustainable Built Environment with the theme Enhancing Disaster Mitigation and Prevention, May 25-27, 2010, Jogjakarta, Indonesia.

Provincial Government of Bulacan. (2007). About Bulacan. Retrieved from http://www.bulacan.gov.ph/generalinfo/physicalprofile.php.

Rahman, H. (2002). Community-based disaster information management system: perspective Bangladesh, Regional Workshop on Best Practices in Disaster Mitigation, Asian Disaster Preparedness Center.

Sy, M. (2010). Disaster risk reduction, management law signed, The Philippine Star, June 10, 2010. Retrieved from http://www.philstar.com/Article.aspx?articleid=583034.

Velez, F. (2010). Bulacan bags Gawad Kalasag 'Hall of Fame’, Manila Bulletin, October 11, 2010. Retrieved from http://www.mb.com.ph/articles/281684/bulacan-bags-gawad-kalasag-hallfame.

Watanabe. M. (no date). Building a tougher disaster coping capacity, Institute for International Disaster Prevenion and Peace Inc. 